\title{
DNAH9 Gene
}

National Cancer Institute

\section{Source}

National Cancer Institute. DNAH9 Gene. NCI Thesaurus. Code C150218.

This gene is involved in cilium movement. 